Dorota Karwacka-Pastor

Facoltà di Filologia

Università di Danzica

Istituto di Filologia Romanza

Dipartimento di Letterature Romanze

https://doi.org/10.18778/8220-478-0.11

\title{
ITALIANITÀ NEGATIVA NELLA LETTERATURA E NELLA PSICOLOGIA
}

\begin{abstract}
L'Italia è stata criticata da poeti e scrittori italiani del passato. La vedevano divisa, piena di caos, vendetta, violenza e odio reciproco tra le sue regioni. Si condannavano e denunciavano l'egoismo, il degrado morale, le iniquità, le scelte sbagliate. Oggi gli intellettuali italiani sono preoccupati: considerano l'Italia un paese malato, corrotto, infelice, dove non si rispetta il bene comune. Danno la colpa alla mentalità italiana e alla mancata solidarietà fra i cittadini.
\end{abstract}

Parole chiave: Italia, italiani, scrittori, letteratura, mentalità, giudizio

Abstract: Italy was criticised by the Italian poets and writers of the past. It was perceived as a divided country, ruled by chaos, revenge, violence and mutual hatred. They condemned egoism, moral degradation, iniquity, wrong choices. Nowadays Italian intellectuals are sounding the alarm, stating that Italy is a sick, corrupted and unhappy country, with no respect for the common good. The main reason for this is Italian mentality and the lack of solidarity among the citizens.

Keywords: Italy, Italians, writers, literature, mentality, view

Questo popolo di santi, di poeti, di navigatori, di nipoti, di cognati...

Ennio Flaiano

L'Italia è un paese pronto a piegarsi ai peggiori governi. È un paese dove tutto funziona male, come si sa. Ė un paese dove regna il disordine, il cinismo, l'incompetenza, la confusione. E tuttavia, per le strade, si sente circolare l'intelligenza, come un vivido sangue. Ė un'intelligenza che, evidentemente, non serve a nulla.

Essa non è spesa a beneficio di alcuna istituzione che possa migliorare di un poco la condizione umana. Tuttavia scalda il cuore e lo consola, se pure si tratta d'un ingannevole, e forse insensato, conforto.

Natalia Ginzburg 
L'italianità è una nozione affascinante e controversa; racchiude in sé sia concetti negativi del modo di essere italiani, che quelli positivi. I costumi, la mentalità, lo stile di vita degli abitanti del Belpaese sono stati molte volte lodati e ammirati, derisi e criticati nella letteratura, nell'arte e nel cinema. Le voci sono state molte e di tonalità differenti, in questo ultimo giro di secoli. I grandi italiani del passato hanno trovato l'Italia divisa e infelice. Non solo Dante, Petrarca, Machiavelli e Leopardi condannavano degrado morale e iniquità dei loro contemporanei, l'hanno fatto anche gli scrittori recenti. Perfino oggi gli studiosi e intellettuali italiani si dicono preoccupati, considerando l'Italia un paese malato. Danno la colpa alla mentalità italiana, al passato doloroso e alla mancata solidarietà fra gli italiani.

A partire dal Purgatorio dantesco, dove troviamo un giudizio negativo sugli italiani scostumati e malgovernati, attraverso il componimento CXXVIII del Canzoniere, Italia mia, benché 'l parlar sia indarno di Petrarca, dove viene stigmatizzata la lotta fratricida nella Penisola, arriviamo alle Seniles rispecchianti la delusione e il ribrezzo del poeta verso i costumi italiani ${ }^{1}$. Secondo il parere di Petrarca, la sete di gloria e di lusso, l'egocentrismo portato all'estremo, l'avidità e l'avarizia, la superbia, la mancanza di scrupoli caratterizzavano gli italiani della sua epoca. Il mondo descritto nelle Seniles è un mondo dominato dagli usurai disonesti, dai giudici corrotti, dai falsi testimoni, dai traditori. Attivi sono i malvagi, i poveri non hanno voce, gli ingenui vengono sfruttati senza pietà.

Nel Rinascimento la situazione nella Penisola non cambia, anzi, sembra peggiorare. Lo testimonia Machiavelli, il quale non esita ad elencare numerosi errori politici e militari commessi dai governanti italiani. Nel Principe e nelle Istorie Fiorentine, egli dipinge un quadro drammatico dell'Italia invasa, tradita, lacerata, odiata dagli stessi cittadini ${ }^{2}$. Essi sono la causa della rovina ${ }^{3}$. La loro spensieratezza e l'orgoglio, la voglia di dominare e la litigiosità rendono gli italiani incapaci, inefficaci, deboli. L'Italia diventa una preda facile per gli invasori. Gli italiani vivono "in pianto", disperati, ma arrabbiati, vendicativi e poco leali fra di loro. Non riescono ad imparare a come diventare fratelli, eppure sanno che per difendersi da pericoli esterni bisogna agire insieme. Ecco la quintessenza dell'italianità negativa, uno sguardo acuto e preoccupato,

1 Nelle Seniles Petrarca indica i vizi e i peccati degli italiani dei suoi tempi. Sono innanzitutto: la voluttà incontrollata, gli stupri, gli adulteri, gli incesti, le frodi, gli inganni, le manipolazioni, i furti, le rapine, le calunnie. Particolarmente interessante è la Lettera III del 1373 destinata a Lombardo da Serico. F. Petrarca (1869), Lettere Senili di Francesco Petrarca, Firenze, Le Monnier, pp. 1161-1162.

2 N. Machiavelli (1954), Opere, Milano-Napoli, Riccardo Ricciardi, pp. 567-568.

3 Ibidem, p. 948. 
una verità conosciuta da tutti, ma detta finalmente con la durezza necessaria. L'Italia sta per morire, vuole dire Machiavelli, anzi, le sue province "sono già morte" 4 .

Nemmeno Leopardi risparmia agli italiani una critica spietata. Nel Discorso sopra lo stato presente dei costumi degl'Italiani, definisce la radice dei mali italiani: è l'assenza della "società stretta". Gli italiani, essendo da sempre nemici, non capiscono l'idea della società, per cui sono privi "d'ogni fondamento di morale, e d'ogni vero vincolo e principio conservatore della società"'. Un altro problema costituisce il carattere nazionale degli italiani, il quale, a parere di Leopardi, non può servire da esempio a nessuno. Gli abitanti del Belpaese sono spensierati, frivoli, pigri e adorano i divertimenti facili, trascurando il lato spirituale ${ }^{6}$. Non sanno inoltre aiutarsi a vicenda né darsi un appoggio reciproco. Risultano indifferenti nei confronti del prossimo, poco empatici, a volte cinici ${ }^{7}$. Gli piace criticare gli altri, sparlare, spettegolare, deridere ${ }^{8}$. Tutto ciò porta all'odio verso chi non è come loro, con loro, per loro. Negli occhi di Leopardi è un'attitudine pericolosa e dannosa che aliena, divide, discrimina. Eppure, criticando gli altri, gli italiani non accettano la critica nei loro confronti, in quanto sono permalosi e vulnerabili. Si considerano degni di lode e di rispetto. Tuttavia, sono completamente inaffidabili, poiché agiscono guidati dall'istinto incontrollabile, dalle passioni negative, quali ira, avidità, desiderio, disperazione, invidia 9

Alfio Squillaci nota, ritornando al pensiero leopardiano:

Prima dunque che Giacomo Leopardi prendesse la penna per dirci in prosa, brutalmente, come siamo fatti, la percezione del carattere dell'italiano aveva occupato l'ingegno di molti letterati europei di prim'ordine e tenuto desto lo spirito di osservazione di una fitta schiera di viaggiatori che, tra Sei e Settecento, giungevano nel Bel Paese col proposito di completare la propria formazione "classica" grazie alla formidabile esperienza del Grand tour [...]. Ma se partivano costoro con programmi culturali di sopralluoghi fra capitelli abbattuti e colonne smozzicate del Foro romano, soprattutto

4 Ibidem, p. 949.

5 G. Leopardi (2010), Discorso sopra lo stato presente dei costumi degl'Italiani, Prato, Piano B Edizioni, p. 28.

6 “[... la vivacità del carattere italiano $[\ldots]$ fa loro preferire i piaceri degli spettacoli e gli altri diletti de' sensi a quelli più particolarmente propri dello spirito, e che gli spinge all'assoluto divertimento scompagnato da ogni fatica dell'animo e alla negligenza e pigrizia [L,]" (Ibidem).

7 Lo stesso dice oggi Luca Fontana sugli italiani contemporanei. L. Fontana (2014), Il costume degli italiani. Breviario di vizi, Parma, Fedelo's Editrice, p. 16.

8 G. Leopardi (2010), op. cit., pp. 40-41.

9 Ibidem, pp. 18-19. 
se ne tornavano in patria, atterriti e soddisfatti, dopo passaggi perigliosi tra lerce locande ed emozionanti agguati di briganti, con taccuini pieni di massime antropologiche come quella del Grosley: «L'Italie est le pays où le mot furbo est éloge». Partivano dalle loro fredde plaghe con l'occhio esaltato dei bellettristi e tornavano con il regard éloigné degli antropologi, e l'Italia e gli italiani nei loro resoconti servivano da formidabile banco di prova per la nascente antropologia culturale, per la letteratura comparata, per la Kulturkritik. Cercavano la classicità e trovavano les sauvages de l'Europe, frugavano fra le sembianze degli italiani dell'oggi alla ricerca dei lineamenti, culturali soprattutto, dei loro antenati, le tracce delle prische virtù romane e i segni forti della civiltà Comunale e del Rinascimento, e non trovavano che un impasto di erotismo, crimine e devozione. Intentavano un viaggio nello spazio e ne facevano invece uno nel tempo: più che andare in giù, nach dem Süden come Goethe, scivolavano lungo il pendio della storia, compiendo un viaggio nell'arretratezza socio-culturale di un popolo e di un Paese che dopo il Rinascimento aveva visto impaludarsi, come i propri fiumi e i propri porti abbandonati, le correnti di civiltà (che erano poi serpeggiate in tutta Europa), nelle forme immobili e pietrificate del proprio essere. Un popolo notevole per ciò che era stato e che poteva essere ma non per ciò che in effetti era. Che aveva un passato e forse un futuro, ma non un presente. Destinato comunque a subire la condanna di sempre degli italiani, quella pronosticatele molti anni dopo dal Generale de Gaulle, e cioè di passare da "Pays pauvre" a "pauvre Pays"!

Trovavano un'Italia passionale e sanguinaria, elegante e crudele, colta e decadente, in ritardo sulla storia ma in anticipo sulle passioni, sempre alle prese con un repentino balenare di pugnali e un sinistro e silenzioso operare di veleni. Ed era proprio questa Italia, questo mito romantico, dove l'uomo non è che sensazione, voluttà, energia, brio e assassinio $[\ldots]^{10}$.

Alcuni scrittori del Novecento hanno osato esprimere la critica verso i loro connazionali in un modo abbastanza brutale. Basta leggere il Giornale di guerra e di prigionia di Carlo Emilio Gadda, il quale condanna la "discordia nella vita nazionale", l'imbecillità" e le "frivolezze" sia nell'esercito italiano, che nella politica, per capire la loro amarezza e la delusione. Lo scrittore aggiunge non senza tristezza: "La nostra anima stupida, porca, cagna, bastarda, superficiale, asinesca tiene per dignità personale il dire: «io faccio quello che voglio», non ho padroni. Questo si chiama fierezza, libertà, dignità" ${ }^{11}$.

10 La Frusta Letteraria, Rivista di critica culturale on line, Squillaci Alfio, Postfazione al Discorso sopra lo stato presente dei costumi degli italiani di Giacomo Leopardi, www.lafrusta.net/riv_italiani.html [29/02/2020].

11 C. E. Gadda (1992), Giornali di guerra e di prigionia, [in:] Saggi, giornali, favole ed altri scritti II, a cura di C. Vela ed al., vol IV, Milano, Garzanti, p. 578. 
In un altro brano Gadda dà voce alla delusione e alla rabbia, utilizzando le parole dure, crudeli, esagerate:

Che porca rabbia, che porchi italiani. Quand'è che i miei luridi compatrioti di tutte le classi, di tutti i ceti, impareranno a tener ordinato il proprio tavolino da lavoro? a non ammonticchiarvi le carte d'ufficio insieme alle lettere della mantenuta, insieme al cestino della merenda, insieme al ritratto della propria nipotina, insieme al giornale, insieme all'ultimo romanzo, all'orario delle Ferrovie, alle ricevute del calzolaio, alla carta per pulirsi il culo, al cappello sgocciolante, alle forbici delle unghie, al portafogli privato, al calendario fantasia? Quando, quando? Quand'è che questa razza di maiali, di porci, di esseri capaci soltanto di imbruttire il mondo col disordine e con la prolissità dei loro atti sconclusionati, proverrà alle attitudini dell'ideatore e del costruttore, sarà capace di dare al seguito delle proprie azioni un legame logico? ${ }^{12}$.

La critica da parte degli intellettuali prosegue nel secondo Novecento e anche dopo l'anno 2000. Giuseppe Prezzolini nell'Italia fragile denuncia la situazione insopportabile del suo paese. Vede l'Italia amministrata male e corrotta. Ci regnano la falsità e l'avversione reciproca tra i politici, generali, intellettuali, cittadini medi. Nulla è sicuro, tutto è possibile, anche gli scenari peggiori ${ }^{13}$.

Gli studiosi, i letterati, i giornalisti definiscono gli italiani superficiali e disonesti. A parere di Luca Goldoni (Italia veniale. Viaggio fra i peccati nazionali) gli italiani sono irresponsabili e insolenti. Per Giuseppe De Rita e Antonio Galdo (Il popolo e gli dei) l'istruzione e la cultura non contano più in Italia ${ }^{14}$. Il ruolo importante gioca il divismo. Tutti o quasi tutti sono narcisi, ognuno vuole raggiungere successo, spesso grazie all'immagine manipolata. Conta troppo l'aspetto, poco lo spirito ${ }^{15}$.

Luigi Barzini nel libro Gli italiani condanna il caos in Italia. Lamenta l'esagerata drammatizzazione in Italia e l'adulazione. Condanna il culto del denaro e la mancanza di vergogna nel commettere i reati, quali truffe, speculazioni fraudolente, inganni di ogni tipo ${ }^{16}$.

12 Ibidem, p. 574.

I brani sono stati tratti dal Giornale di guerra e di prigionia di Carlo Emilio Gadda e sono datati per il 24 luglio 1916 e il 31 luglio 1916.

13 G. Prezzolini (1974), L'Italia fragile, Milano, Pan Editrice.

14 A. G. e C. De Rita (2014), Il popolo e gli dei. Cosi la grande crisi ha separato gli italiani, Roma-Bari, Laterza; L. Goldoni (1973), Italia veniale. Viaggio fra i peccati nazionali, Bologna, Cappelli.

15 Ibidem, p. 63.

16 L. Barzini (2015), Gli italiani. Virtù e vizi di un popolo, Milano, Rizzoli, pp. 118-121, 129, 139-140. 
Raffaele Caserta scrive: "uno dei luoghi comuni più abusati afferma che siamo un popolo di eroi, santi, poeti e navigatori e ciò ha sempre solleticato il nostro orgoglio nazionale"17, "ma" - aggiunge subito l'autore - "forse sarebbe per noi italiani più adatto il famoso detto del commediografo Bertold Brecht: Beato quel paese che non ha bisogno di eroi". Elenca poi i tratti caratteristici della mentalità italiana, tra cui il primo è la sudditanza psicologica verso il potere. Parla anche dell'individualismo esasperato che comporta la mancanza assoluta di senso organizzativo in tutti i settori della vita sociale. Emblematica è anche, secondo l'autore, la ricerca di personalità di tipo messianico, dell'"uomo della provvidenza" (Mussolini, Berlusconi).

Gli italiani, dice Caserta esattamente come Leopardi, non possiedono il senso dello Stato, non si sentono parte di una comunità nazionale. Inoltre, gli manca l'etica, non credono nei valori morali privati né professionali. Un grande valore diventa invece l'arte di arrangiarsi, la furbizia: "in pratica è sempre privilegiato chi è furbo rispetto a chi è intelligente" 18 .

Lautore nota la mancanza di rispetto e stima per il patrimonio culturale:

L'ignoranza culturale è un'altra caratteristica grave in un popolo che sul suo territorio vanta il sessanta-settanta per cento dei monumenti artistici mondiali, ma non riesce a fruirne che in modo ridottissimo, per le chiusure museali nei giorni festivi o quantomeno nei giorni od ore di più tempo libero, oltre al fatto che sono più gli stranieri ad interessarsi alle nostre bellezze artistiche che gli italiani ${ }^{19}$.

Vittorino Andreoli, uno dei più famosi psichiatri italiani ${ }^{20}$, dà una diagnosi drammatica, definendo l'Italia un paese profondamente malato, impossibile

17 AgoraVox Italia, Caserta, Raffaele, Aspetti negativi del carattere degli italiani agoravox.it/Aspetti-negativi-del-carattere.html [29/02/2020].

18 Ne parla anche Prezzolini: "L'Italiano ha un tale culto per la furbizia, che arriva persino all'ammirazione di chi se ne serve a suo danno. Il furbo è in alto in Italia non soltanto per la propria furbizia, ma per la reverenza che l'italiano in generale ha della furbizia stessa, alla quale principalmente fa appello per la riscossa e per la vendetta. Nella famiglia, nella scuola, nelle carriere, l'esempio e la dottrina corrente - che non si trova nei libri - insegnano i sistemi della furbizia. La vittima si lamenta della furbizia che l'ha colpita, ma in cuor suo si ripromette di imparare la lezione per un'altra occasione". G. Prezzolini (1974), op. cit., p. 41.

19 AgoraVox Italia, Caserta, Raffaele, Aspetti negativi del carattere degli italiani www.agoravox.it/Aspetti-negativi-del-carattere.html [29/02/2020].

20 Ex direttore del Dipartimento di psichiatria di Verona, membro della New York Academy of Sciences e presidente del Section Committee on Psychopathology of Expression della World Psychiatric Association. 
da salvare ${ }^{21}$. Nel libro intitolato Ma siamo matti. Un paese sospeso fra normalità e follia, Andreoli indica alcuni sintomi di questa misteriosa malattia italiana. Sono gli stessi mali presenti in Italia da sempre, già definiti, descritti e analizzati dagli scrittori delle epoche passate. Essi dominano la mente e il cuore della popolazione della Penisola. Lo psichiatra elenca l'odio, l'antipatia, la voglia di affermarsi ad ogni costo e di avere sempre ragione. Gli italiani sono, a suo parere, individualisti guidati dall'egoismo, ipocriti e invidiosi. Sono conformisti. Non rispettano il bene pubblico. Gli manca la vergogna. Sono pronti a distruggere chi è il loro rivale, incapaci di collaborare in modo pacifico e rispettoso per il bene comune $e^{22}$.

Lo psichiatra li vede anche indecisi, distratti, menefreghisti. Il rinvio è diventato simbolo della vita italiana: non fare mai oggi quello che potresti fare domani. È importante in Italia non compromettersi, rimandare la scelta; fare il doppio gioco.

Per Andreoli gli italiani sono "distruttori di loro stessi, alla ricerca di un rapporto di disprezzo e di dominio", commettono "scelte dannose", forse perché sono stati sottomessi a tanti invasori, "soggetti a tutti i popoli egemoni della storia" ${ }^{23}$. Sono, inoltre, il popolo creativamente più ricco che non sa e non fa abbastanza per conservare e apprezzarle le ricchezze culturali che possiede. In Italia regna il caos, eppure tutti portano "una maschera allegra" nonostate la crisi economica e familiare ${ }^{24}$.

Lo studioso nota problemi sociali, di comunicazione e di collaborazione ("l'italiano è senza dimensione del noi"). Eppure "Il noi è vita, mentre l'io è morte [...]. Insomma, la nostra dimensione è il noi". Sfortunatamente "oggi raramente si ricorre all'uso del «noi», con una netta predominanza dell' «io», e ancora più raramente ci si avvale del termine «popolo»"25.

Lo psichiatra annuncia la morte della morale ${ }^{26}$ : "il popolo italiano ha incorporato inconsapevolmente la concezione della morale come utile

21 Andreoli dice: "Io sono convinto che il popolo italiano sia malato. [...] è proprio l'amore a rendermi critico [...]”. V. Andreoli (2015), Ma siamo matti. Un paese sospeso fra normalità e follia, Milano, Rizzoli, pp. 12, 14.

22 Ibidem, pp. 22, 27, 35, 42.

23 Ibidem, p. 21.

24 Ibidem, p. 27.

25 Ibidem, pp. 3-4, 48.

26 “[... ] c'è l'io in famiglia, c’è l'io dappertutto. Siamo dei narcisi spaventosi, tutto io, faccio io. [...] Pur di essere «io» faccio qualsiasi cosa, è un narcisismo fondato sull'io e sul mio. Quello che è del «noi» non importa, lo roviniamo. Questo, in un momento in cui si dovrebbe costruire qualcosa insieme, ognuno suona il proprio strumento, come se lei ascoltasse un'orchestra dove ognuno suona per conto suo. Ė un delirio dell'io. È una grave malattia [... ]”. Il brano sucitato è stato tratto dall'intervista 
a «me» e al «mio», il resto non lo riguarda, al massimo condanna come maleducato $[\ldots]^{27}$.

La parola è fondamentale in Italia, poiché "il popolo italiano vive di parole, è gratificato dalle parole" 28 , ma la parola viene usata male, "in modo spaventosamente esagerato". Un altro problema è che "è più importante il dire che il fare" 29 . È troppo frequente, poi, la critica dell'interlocutore, l'imporre il proprio parere e la menzogna. In più, l'italiano ama la maldicenza in modo appassionato ${ }^{30}$, e tutto ciò nasce dalla frustrazione.

Andreoli condanna anche il mammismo come particolarmente nocivo ${ }^{31}$ e il narcisismo come ridicolo e pericoloso. Gli italiani pensano di possedere doti straordinarie, di aver diritto a continui riconoscimenti, e se ciò non succede, scatta l'odio per i mancati estimatori. Lo studioso denuncia anche l'onnipresente ossessione dei vestiti firmati e delle spese, e incita i lettori a ricordare che "occorre mettere al primo piano la persona e, nell'insieme, il popolo, e poi preoccuparsi di quel che fa apparire affascinanti, interessanti e persino belli, non dimenticando che il riferimento deve essere l'uomo o la donna e non l'oggetto indossato" ${ }^{2}$. La soluzione per guarire è, fra l'altro, "togliere al denaro il potere che gli abbiamo dato, come misura di tutte le cose, il denaro ha assunto una dimensione enorme $[\ldots]]^{\prime 3}$.

Gli italiani sono passivi, si lamenta il profesor Andreoli, a causa della "fede nel miracolo" che non succede mai, ma che permette loro di fare niente, di non provare a cambiare la realtà:

Siamo un popolo di creduloni, che aspettano il "miracolo" che risolverà i nostri problemi. E poi siamo un popolo che ha la fortuna, il destino, l'oroscopo, i maghi, i gratta e vinci. Poi siamo pieni di patroni, si nomina sempre San Gennaro, ma a Verona c’è San Zeno, siamo pieni di questi patroni che ci fanno andare bene le $\operatorname{cose}^{34}$.

con Andrea Purgatori apparsa il 15 agosto 2015. RaiNews, L'Italia è matta? Intervista di Pierluigi Mele a Vittorino Andreoli: confini.blog.rainews.it/2015/03/21/litalia-ematta-intervista-a-vittorino-andreoli/ [29/02/2020].

27 V. Andreoli (2015), op. cit., p. 41.

28 Ibidem, p. 62.

29 Ibidem, p. 55.

30 Ibidem, p. 101.

31 Ibidem, p. 125.

32 Ibidem, p. 140.

33 RaiNews, L'Italia è matta? Intervista di Pierluigi Mele a Vittorino Andreoli confini.blog.rainews.it/2015/03/21/litalia-e-matta-intervista-a-vittorino-andreoli/ [29/02/2020]

34 Ibidem. 
Lo psichiatra, come anche altri autori, intellettuali e osservatori della vita sociale italiana, menziona un altro tratto molto caratteristico della mentalità italiana che è l'arte di arrangiarsi, elemento tipico, in quanto "gli italiani hanno inventato da secoli antiche astuzie per sconfiggere il tedio e la disciplina, per dimenticare l'umiliazione e la sfortuna: di questo sono maestri" 35 .

Invece Alessandro Campi nel libro Congiure e complotti. Da Machiavelli a Beppe Grillo $^{36}$ definisce l'Italia "malata di complottismo e dietrologia" ${ }^{37}$. Nella sua opinione gli italiani sono sospettosi, superstiziosi, diffidenti. Sono assolutamente ossessionati dal complottismo perché è consolatorio, appagante e gratificante. È il segno di "una regressione dell'intelligenza, che ha perduto la forza di pensare la realtà. E ciò comporta "una cultura del sospetto permanente, una diffidenza costante nei confronti delle istituzioni che rischia di essere letale per la vita civile di qualunque Paese". Lo studioso definisce la mentalità complottista italiana come "un misto di paranoia e sospetto"38.

Gli italiani di oggi sono rassegnati, in Italia regna l'apatia generale, la mancanza di solidarietà e di una iniziativa comune. Ne parla Giorgio Bocca nella sua Italia l'è malada dicendo che l'italiano è "stanco di non capire, di essere preso in giro, del dire e disdire, delle menzogne plateali, del cattivo gusto che monta; stanco della vecchia, vecchissima storia dei furbi che derubano gli onesti e gli ingenui" 39 .

Nel libro Caduti in corsia: viaggio in un'Italia malata del 2011 Michele Iula definisce l'Italia una repubblica fondata sulla malasanità e sulla corruzione. Nota che gli italiani si perseguitano vicendevolmente, deridono l'un l'altro, "si pungono fino al sangue, mostrando con le parole e coi modi ogni sorta di disprezzo verso l'avversario politico" 40 .

È difficile - dicono coloro che si definiscono preoccupati per l'Italia e per gli italiani - trovare un rimedio a questo drammatico e pericoloso stato di cose. Ci sono tante proposte e parecchie vie, ma non c’è la forza per cambiare né per unirsi o correggersi.

\section{Ibidem.}

Luca Barzini scrive a proposito che "l'arte di vivere, quest'arte screditata" è stata "creata dagli italiani per sconfiggere l’angoscia e la noia" (Barzini 2015: 433).

36 Alesandro Campi è docente di Storia delle dottrine politiche, Scienza politica e Relazioni internazionali dell'Università di Perugia.

37 A. Campi (2016), Congiure e complotti. Da Machiavelli a Beppe Grillo, Soveria Mannelli, Rubbettino, p. 72.

38 Ibidem, p. 75.

39 G. Bocca (2005), Italia l'è malada, Milano, Feltrinelli, p. 32.

40 M. Iula (2011), Caduti in corsia: viaggio in un'Italia malata, Roma, Editori Internazionali Riuniti, p. 54. 
Tuttavia, nonostante l'italianità negativa, nonostante tante voci critiche, il Belpaese rimane la patria dell'arte, della cultura e dell'ispirazione, con un passato storicamente doloroso, ma artisticamente glorioso. Ė sempre la stessa Terra promessa, amata e desiderata dagli intellettuali europei delle epoche passate che sognavano di mandare i loro figli a studiare presso le università italiane. Ora l'Italia è indubbiamente in crisi, come affermano unanimemente tanti studiosi italiani. Eppure non tutto è perduto e dagli italiani stessi dipende il loro avvenire. Giuste e saggie paiono le parole di Raffaele Caserta, il quale spera di vedere un futuro migliore per l'Italia:

Questi fattori possono farci regredire un una decadenza irreversibile, in cui il nostro pregio più rilevante è una grande pazienza capace di farci sopravvivere a tanto squallore. Poiché la storia è fatta di cicli temporali positivi e negativi ci può confortare che forse i nostri difetti non sono atavici o genetici ma rimediabili in un prossimo futuro dove i nostri discendenti vedranno l'alba di un nuovo Rinascimento ${ }^{41}$.

\section{Bibliografia}

Alighieri, Dante (2010). Divina Commedia, Purgatorio, Roma, Newton Compton.

Andreoli, Vittorino (2015). Ma siamo matti. Un paese sospeso fra normalità e follia, Milano,

Rizzoli.

Barzini, Luigi (2015). Gli italiani. Virtù e vizi di un popolo, Milano, Rizzoli.

Bocca, Giorgio (2005). Italia l'è malada, Milano, Feltrinelli.

Bollati, Giulio (2011). L'italiano. Il carattere nazionale come storia e come invenzione, Torino, Einaudi.

Campi, Alessandro (2016). Congiure e complotti. Da Machiavelli a Beppe Grillo, Soveria Mannelli, Rubbettino.

De Rita, Giuseppe e Galdo, Antonio (2014). Il popolo e gli dei. Così la grande crisi ha separato gli italiani, Roma-Bari, Laterza.

Fontana, Luca (2014). Il costume degli italiani. Breviario di vizi, Parma, Fedelo's Editrice.

Gadda, Carlo Emilio (1992). Giornali di guerra e di prigionia, [in:] Saggi, giornali, favole ed altri scritti II, a cura di C. Vela ed al., vol. IV, Milano, Garzanti.

Goldoni, Luca (1973). Italia veniale. Viaggio fra i peccati nazionali, Bologna, Cappelli.

Iula, Michele (2011). Caduti in corsia: viaggio in un'Italia malata, Roma, Editori Internazionali Riuniti.

Leopardi, Giacomo (2010). Discorso sopra lo stato presente dei costumi degl'italiani, Prato, Piano B Edizioni.

Machiavelli, Niccolò (1954). Opere, Milano-Napoli, Riccardo Ricciardi.

Machiavelli, Niccolò (2000). Scritti politici, Milano, Mursia.

Petrarca, Francesco (2010). Canzoniere, Milano, Mondadori.

Petrarca, Francesco (1869). Lettere Senili di Francesco Petrarca, Firenze, Le Monnier.

Prezzolini, Giuseppe (1974). L'Italia fragile, Milano, Pan Editrice.

41 AgoraVox Italia, Caserta, Raffaele, Aspetti negativi del carattere degli italiani www.agoravox.it/Aspetti-negativi-del-carattere.html [29/02/2020]. 


\section{Sitografia}

RaiNews, L'Italia è matta? Intervista di Pierluigi Mele a Vittorino Andreoli confini.blog.rainews. it/2015/03/21/litalia-e-matta-intervista-a-vittorino-andreoli/ [29/02/2020].

AgoraVox Italia, Caserta Raffaele, Aspetti negativi del carattere degli italiani www.agoravox.it/ Aspetti-negativi-del-carattere.html [29/02/2020].

La Frusta Letteraria, Rivista di critica culturale on line, Squillaci Alfio, Postfazione al Discorso sopra lo stato presente dei costumi degli italiani di Giacomo Leopardi www.lafrusta.net/ riv_italiani.html [29/02/2020].

L'opinione delle libertà, Abbate Mario Scaffidi, L'Italia, l'eterna ammalata www.opinione.it/ cultura/2013/02/20/scaffidi_cultura-20-02 [29/02/2020]. 